\title{
Genotypic Analysis Revealed Association of HLA Alleles with Clinical Parameters in Bangladeshi Patients with Rheumatoid Arthritis
}

\author{
Fauzun Shaona', Rubyyat Hassan ${ }^{2}$, Sajib Chakraborty' ${ }^{1}$, Shahanaz Sultana', Jobaida Akther ${ }^{1}$, \\ AHM Nurun Nabi ${ }^{*}$ \\ ${ }^{1}$ Laboratory of Population Genetics, Department of Biochemistry and Molecular Biology, University of Dhaka, Dhaka, Bangladesh \\ ${ }^{2}$ Popular Diagnostic Center, Dhanmondi Branch, Dhaka, Bangladesh \\ Email: *nabi@du.ac.bd
}

How to cite this paper: Shaona, F., Hassan, R., Chakraborty, S., Sultana, S., Akther, J. and Nabi, A.H.M.N. (2019) Genotypic Analysis Revealed Association of HLA Alleles with Clinical Parameters in Bangladeshi Patients with Rheumatoid Arthritis. American Journal of Molecular Biology, 9, 173-190.

https://doi.org/10.4236/ajmb.2019.94013

Received: June 16, 2019

Accepted: September 14, 2019

Published: September 17, 2019

Copyright $\odot 2019$ by author(s) and Scientific Research Publishing Inc. This work is licensed under the Creative Commons Attribution International License (CC BY 4.0).

http://creativecommons.org/licenses/by/4.0/

\begin{abstract}
This study investigated distribution of HLA alleles (HLADRB1 ${ }^{\star} 01,{ }^{\star} 03,{ }^{\star} 04$, $\left.{ }^{\star} 07, \mathrm{HLA}-\mathrm{DQB} 1{ }^{\star} 0201,{ }^{*} 0301 / 4\right)$ in 34 healthy controls and 57 rheumatoid arthritis (RA) patients in a Bangladeshi population and correlated the genotypic frequencies with clinical parameters. Frequency distribution of HLADRB ${ }^{\star} 04(34 \%)$ and HLA-DRB1*01 (32\%) were the highest followed by HLADQB1 ${ }^{\star} 0301 / 4(29 \%)$ and HLA-DQB1 ${ }^{\star} 0201(26 \%)$ in RA patients while HLA$\mathrm{DRB}^{*} 03(12 \%)$ had lowest frequency. Plasma level of anti-CCP and rheumatoid factor antibodies confirmed diagnosis of RA patients that varied significantly between patients and healthy controls. Likewise, plasma levels of C-reactive protein, triglycerides, cholesterol, HDL-cholesterol and activities of AST and ALT exhibited significant variation between the two groups. In contrast, the levels of glucose, total protein, uric acid, LDL-cholesterol and plasma activity of ALP in RA patients had no significant deviations from healthy controls. Relationship between HLA genotype frequency and clinical parameters revealed that the mean levels of anti-CCP and rheumatoid factor antibodies were highest in the patients harboring HLA-DRB1 ${ }^{\star} 04$ allele. These findings underpin the correlation between HLA genotype with clinical markers of RA which are indicative of disease severity. The positive correlation of these markers with certain HLA genes may be used to identify susceptible individuals who are likely to have RA in Bangladeshi population.
\end{abstract}

\section{Keywords}

Rheumatoid Arthritis, HLA Allele, Bangladeshi Population, Gene Polymorphisms, Genotypes 


\section{Introduction}

Rheumatoid arthritis (RA) is characterized by chronic inflammation caused by autoimmune disease attacking joints that leads to pain, swelling, stiffness and eventual destruction of joints. RA is thought to be influenced by both genetic predisposition and environmental factors. Based on numerous studies focusing on the molecular basis and genetic predisposition to uncover the risk-factors of RA, it has been well surmised that high-risk genetic context combined with epigenetic and environmental factors leads to the manifestation of the RA symptoms [1] [2] [3] [4] [5]. Three factors-genetic-make up, environment and autoimmunity that contributes to the onset and progression of RA, have been termed as the "Bermuda triangle" [1]. In recent years with the advent of new technologies, our understanding of the aberrant immune functions leading to infiltration and inflammation of the synovium has advanced significantly. The exact triggers and their timing that causes the onset of RA remain elusive. The heritable contribution in the form of genetic predisposition in the pathogenesis of RA has been estimated to be about $60 \%$ that including human leukocyte antigens (HLA-DR) and non-HLA genes [2] [3]. It was hypothesized that the genetic factor when combined with certain environmental exposure and lifestyle-related factors such as smoking may trigger the onset of RA by inducing the production of anti-cyclic citrullinated peptide antibody (ACCP) [4].

Despite numerous attempts to uncover the comprehensive genetic basis of RA, only HLA-DRB1 allele was found to be linked with risk of RA beyond any reasonable doubt [5]. Different allelic variations of HLA-DRB1 gene have been linked with RA risk and onset, suggesting a possible role of interaction between T-cell receptor (TCR) and HLA-antigen in the underlying pathological process of RA [6]. In an attempt to uncover the underlying mechanism of certain HLA association with RA, Gregerson et al. proposed a hypothesis called "Shared Epitope (SE)" for the association of different HLA-DRB1 alleles with RA. The study showed an RA associated HLA-DRB1 allele encodes a five amino acid long conserved sequence (QRRAA, RRRAA or QKRAA) that resides within the third hyper variable region (HVR3) of the DRb1 chain. These residues form a part of the helical domain of the antigen-binding site and thus are likely to directly affect the antigen presentation process. The HLA alleles that are shown to encode this conserved amino acid sequence include: DRB $1{ }^{\star} 0401,{ }^{\star} 0404,{ }^{\star} 0405,{ }^{\star} 0408$, ${ }^{\star} 0101,{ }^{\star} 0102,{ }^{\star} 1402,{ }^{\star} 09$ and ${ }^{\star} 1001$ [5]. In different population groups representing different ethnicity the frequency of the HLA alleles that are associated with RA vary significantly [5]. For instance, $\mathrm{DRB1} 1^{\star} 0401$ and ${ }^{\star} 0404$ alleles are frequently associated with RA in Caucasians whereas DRB1*0405 and DRB1*0101 alleles are found to be highly associated with RA in Japanese and in Israeli Jews respectively [5]. However, no such studies have been conducted to untangle the HLA genotyping associated with RA in Bangladesh.

It is apparent that in order to minimize disease progression, early diagnosis is of utmost importance to better manage the RA patients. Unfortunately, the early 
diagnosis of RA is still challenging. Since autoantibodies are directly linked with the onset of autoimmune disease, rheumatoid factor (RF) - an autoantibody that targets the Fc portion of IgG, has been one of the most important markers for the RA diagnosis. However, the non-specificity RF has been a major challenge as $\mathrm{RF}$ levels can also be elevated in patients that are suffering from other diseases e.g., Sjogren's syndrome and type 2 cryoglobulinaemia [6], infection (such as HCV infection) [8] and in elderly persons [7]. Due to limited specificity of RF, around $60 \%-70 \%$ of RA patients are diagnosed as RF positives. Only $11-20 \%$ of RF positive patients suffering from masculoskeletal problems have RA. For these reasons RF factor is considered as a good indicator of disease severity rather than diagnostic value [7]. Another serological marker for RA is anti-cyclic citrullinated peptide (anti-CCP) antibodies that are reactive to the citrulline amino acid and are observed to be elevated in the sera of patients with RA [9]. In contrast to RF, anti-CCP antibody is observed to have increased specificity when it comes to RA diagnosis and has emerged as an alternative maker for RA [10]. Although RF may have lower specificity in RA diagnosis, this particular serological marker has a great value as a predictor of RA severity and thus is considered as prognostic marker for RA [11]. Elevated levels of RF are found to be associated with risk of developing RA [12]. RA patients with elevated RF levels are prone to develop a more aggressive form of the disease. Rheumatoid nodules are commonly observed as a manifestation of the elevated RF in the serum of RA patients [13].

The evidence of HLA association with the risk of developing RA, prompted the question whether HLA polymorphisms have a role in the inflammatory reaction and thus can be used as a risk predictor for the aggressiveness of rheumatoid synovitis. Studies based on retrospective analysis showed that HLA genotyping is indeed associated with aggressiveness of rheumatoid synovitis [6] [14]. Alleles such as HLA-DR $B 1^{\star} 01$ are found to be enriched in RA patients characterized by RF-negative representing a variant of RA known to slowly progress compared to RF positive variant of RA. In contrast HLA-DRB1 ${ }^{\star} 0401$ allele was found to be frequently associated with RA positive variant of RA that is considered as a more aggressive form of the disease [14]. These studies have opened a new window of opportunity to evaluate the risk of developing the aggressive form of RA simply by analyzing the HLA genotypes. Other studies, evaluating the utility of HLA genotyping to predict the progression of RA in prospective manner, claimed that HLA-DRB1 ${ }^{\star} 04$ positive RA patients are susceptible to develop inflammatory activities in the joints at the early stage of the disease that causes subsequent destruction of joints [15]. Although HLA-genotyping holds much promise as a prognostic marker, the precise value of HLA genotyping to predict disease progression is still not clear. In addition, the association of HLA genotyping and the levels of disease progression marker-RF have not been studied previously.

The current study was specifically designed to evaluate the association between HLA genotyping and RA propensity in Bangladeshi population. Moreover, in 
this study we sought to uncover the possible link between enrichment of certain HLA DRB1 alleles in the RA patients with elevated levels of clinical parameters such as RF and anti-CCP antibody. In summary the current study has the potential to shed light into the plausible association between HLA genotyping with RA progression by stratifying the patients based on their HLA-allele frequency and clinical parameters- RF and anti-CCP antibodies.

\section{Methods and Materials}

\subsection{Study Design, Enrollment of Study Participants and Sample Collection}

A schematic diagram representing the workflow of the study is shown in Figure 1. The cohort was established by including 57 suspected rheumatoid arthritis (RA) patients and 34 healthy (control) subjects. Samples were collected from the Popular Diagnostic Center, Dhanmondi Branch, Dhaka, Bangladesh by taking consent from the participants. Healthy controls were university students, teachers and office staffs. This study was approved by the ethical review committee for clinical research with human samples of the department of Biochemistry and Molecular Biology, University of Dhaka, Bangladesh. The inclusion criteria of the possible RA patients were based on clinical presentations of the patients. Anthropometric data including sex, age, height and weight for each of the study participants were recorded in a structured questionnaire. Blood samples were collected from each of healthy control subjects and patients with RA. Collected blood samples were centrifuged at $2000 \mathrm{rpm}$ for 10 minutes and plasma samples were stored in $-80^{\circ}$ if not immediately used for further analysis. Cellular fractions of blood samples were re-suspended with equal volume of sterilized normal saline

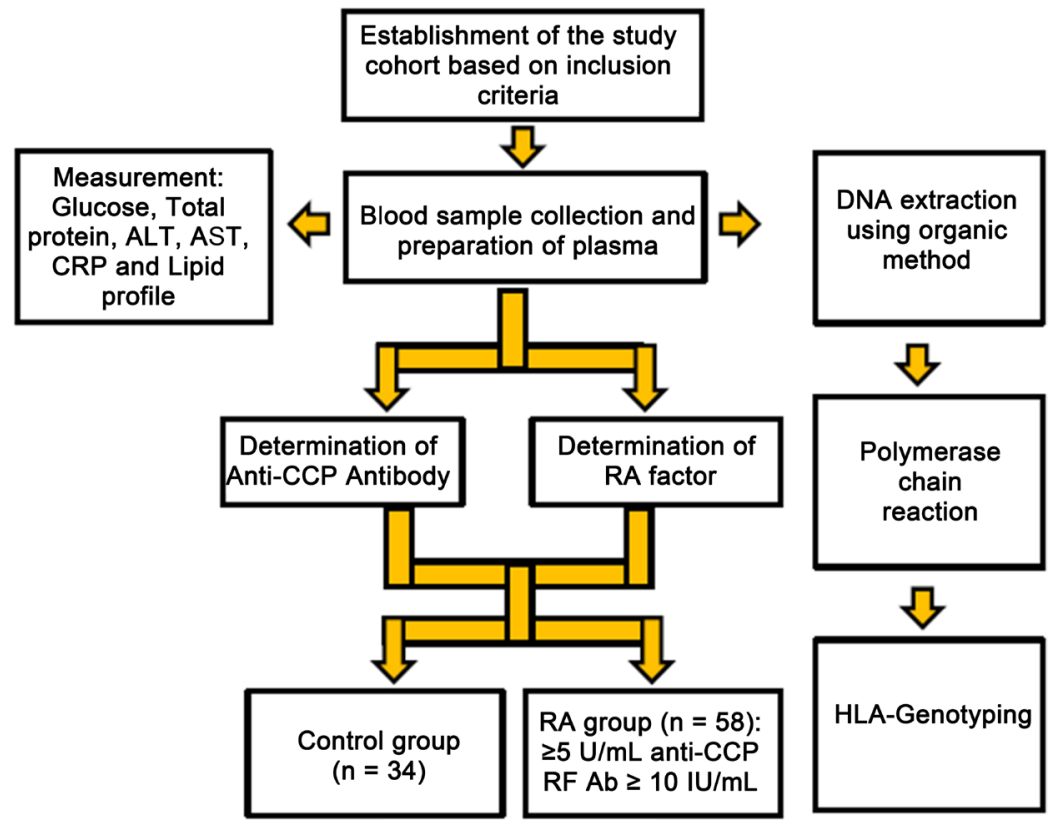

Figure 1. Workflow of the study design, cohort establishment, sample collection, biochemical analyses and HLA-genotyping. 
$(0.9 \%$ of $\mathrm{NaCl})$ and stored at $-80^{\circ} \mathrm{C}$ until extraction of genomic DNA.

\subsection{Determination of the Level of Anti-Cyclic Citrullinated Peptide and Rheumatoid Factor Antibodies}

Anti-cyclic citrullinated peptide (anti-CCP) and RF levels were measured using routine clinical procedures. Briefly, the measurements were carried out by utilizing the immunoassays for the semi-quantitative determination of the IgG class of auto-antibodies specific to cyclic citrullinated peptide (CCP) and RF mediated agglutination in plasma samples using ABBOTT reagent kit.

\subsection{Measurement of Biochemical Parameters}

Plasma levels of c-reactive protein (CRP), total protein, uric acid, cholesterol, triglyceride (TG), high density lipoprotein (HDL)-cholesterol, low density lipoprotein (LDL)-cholesterol, glucose, alanine aminotransferase (ALT), aspartate amino transferase (AST), alkaline phosphatase (ALP) were measured in the plasma samples by the standard clinical procedures.

\subsection{HLA-Genotyping by Polymerase Chain Reaction}

White blood cells of blood samples were used to extract genomic DNA by organic extraction procedure as described by Afruza et al. [16]. All DNA samples were subjected to genotyping for HLA-DRB1 and HLA-DQB1 using PCR-SSP (Polymerase chain reaction with sequence specific primer). A conserved sequence of human growth hormone (HGH) was amplified in reaction as an internal control primer (forward and reverse primer sequences are 5'-GCCTTCCCA ACCATTCCCTTA-3' and 5'-TCACGGATTTCTGTTGTGTTTC-3'). Another conserved sequence of beta-globin was amplified in reaction as another internal control primer; forward and reverse primer sequence are 5'-CAACTTCATCCA CGTTCACC-3' and 5'-GAAGAGCCAAGGACAGTTAC-3'. Specific primer sequences and PCR conditions for determining the frequencies of different HLA alleles are in Table 1.

\subsection{Statistical Analyses}

Demographic data obtained from the structured questionnaire and clinical parameters were analysed using SPSS v21.0 (IBM) in which the results were expressed as mean \pm SD for continuous variables and \% for categorical variables. To compare the differences between different variables from the control and patients, Student's independent t-test was performed using Statistical Package for Social Sciences (SPSS, v21).

\section{Results}

\subsection{Identification of HLA Alleles Using Gel Electrophoresis}

Amplified PCR products representing the distinct HLA alleles were identified according to the band size by gel electrophoresis. For instance, the presence of 
Table 1. Primer sequence and conditions for performing polymerase chain reaction.

\begin{tabular}{|c|c|c|c|}
\hline Gene & Primer sequence & PCR condition & Product size (bp) \\
\hline \multirow[t]{3}{*}{ HLA-DRB1*01 } & FP:5’TTGTGGCAGCTTAAGTTTGAAT3' & $94^{\circ} \mathrm{C}-5 \mathrm{~m}$ & 255 \\
\hline & RP:5'CTGCACTGTGAACGTGTCAC3' & $\left(94^{\circ} \mathrm{C}-30 \mathrm{~s} 51^{\circ} \mathrm{C}-50 \mathrm{~s} 72^{\circ} \mathrm{C}-45 \mathrm{~s}\right) \times 30$ & \\
\hline & & $72^{\circ} \mathrm{C}-5 \mathrm{~m}$ & \\
\hline \multirow[t]{3}{*}{ HLA-DRB $1^{*} 03$} & FR:5’TACTTCCATAACCAGGAGGAGA3' & $95^{\circ} \mathrm{C}-2 \mathrm{~m}$ & 151 \\
\hline & RP:5’TGCAGTACTTGTCCACCCG3' & $\left(95^{\circ} \mathrm{C}-30 \mathrm{~s} 53^{\circ} \mathrm{C}-30 \mathrm{~s} 72^{\circ} \mathrm{C}-25 \mathrm{~s}\right) \times 30$ & \\
\hline & & $72^{\circ} \mathrm{C}-5 \mathrm{~m}$ & \\
\hline \multirow[t]{3}{*}{ HLA-DRB $1^{*} 04$} & FR:5'GTTTCTTGGAGCAGGTTAAACA3' & $95^{\circ} \mathrm{C}-3 \mathrm{~m}$ & 260 \\
\hline & RP:5’CTGCACTGTGAAGCTCTCAC3 & $\left(95^{\circ} \mathrm{C}-30 \mathrm{~s} 53^{\circ} \mathrm{C}-30 \mathrm{~s} 72^{\circ} \mathrm{C}-40 \mathrm{~s}\right) \times 30$ & \\
\hline & & $72^{\circ} \mathrm{C}-5 \mathrm{~m}$ & \\
\hline \multirow[t]{3}{*}{ HLA-DRB1*07 } & FR: 5'CCTGTGGCAGGGTAAGTATA3' & $95^{\circ} \mathrm{C}-2 \mathrm{~m}$ & 232 \\
\hline & RP:5'CCCGTAGTTGTGTCTGCACAC3' & $\left(95^{\circ} \mathrm{C}-30 \mathrm{~s} 53^{\circ} \mathrm{C}-30 \mathrm{~s} 72^{\circ} \mathrm{C}-25 \mathrm{~s}\right) \times 30$ & \\
\hline & & $72^{\circ} \mathrm{C}-5 \mathrm{~m}$ & \\
\hline \multirow[t]{3}{*}{ HLA-DQB1*0201 } & FR:5'GTGCGTCTTGTGAGCAGAAG3' & $95^{\circ} \mathrm{C}-2 \mathrm{~m}$ & 205 \\
\hline & RP:5’GCAAGGTCGTGCGGAGCT3’ & $\left(95^{\circ} \mathrm{C}-30 \mathrm{~s} 53^{\circ} \mathrm{C}-30 \mathrm{~s} 72^{\circ} \mathrm{C}-25 \mathrm{~s}\right) \times 30$ & \\
\hline & & $72^{\circ} \mathrm{C}-5 \mathrm{~m}$ & \\
\hline \multirow[t]{3}{*}{ HLA-DQB1*0301/4 } & FR:5’GACGGAGCGCGTGCGTTA3’ & $95^{\circ} \mathrm{C}-3 \mathrm{~m}$ & 122 \\
\hline & RP:5’AGTACTCGGCGTCAGGCG3' & $\left(95^{\circ} \mathrm{C}-30 \mathrm{~s} 58^{\circ} \mathrm{C}-30 \mathrm{~s} 72^{\circ} \mathrm{C}-25 \mathrm{~s}\right) \times 30$ & \\
\hline & & $72^{\circ} \mathrm{C}-5 \mathrm{~m}$ & \\
\hline
\end{tabular}

$260 \mathrm{bp}$ band indicated that respective individuals were HLA-DRB $1^{*} 04$ positive (Figure 2(a)). Among the 57 RA patients, 20 individuals (34\%) were found to be HLA-DRB1*04 positive whereas only 6 out of 34 healthy controls (17\%) were identified to harbor HLA-DRB $1^{\star} 04$ allele. To investigate the frequency of other HLA alleles among the RA patients the distribution of HLA-DRB $1^{\star} 03$, HLA-DRB1 ${ }^{\star} 01$, HLA-DRB ${ }^{\star} 07$, HLA-DQB ${ }^{\star} 0201$ and HLA-DQB1 ${ }^{\star} 0301 / 4$ alleles were further analyzed. PCR products were analyzed for the presence of HLA-DRB1 ${ }^{\star} 03$ (Figure 2(b)), HLA-DRB1 ${ }^{\star} 01$ (Figure 2(c)), HLA-DRB1 ${ }^{\star} 07$ (Figure 2(d)), HLA-DQB1*0201 (Figure $2(\mathrm{e})$ ) and HLA-DQB1*0301/4 (Figure 2(f)) alleles among the RA patients.

\subsection{Levels of Anti-Cyclic Citrullinated Peptide and Rheumatoid Factor Antibodies in the Plasma of Study Participants}

In order to confirm rheumatoid arthritis, the levels of anti-cyclic citrullinated peptide (anti-CCP) antibody and rheumatoid factor antibody were measured in the plasma of each participant. Higher levels of these antibodies confirmed the diagnosis of rheumatoid arthritis (RA) in patients. All 57 individuals were diagnosed with rheumatoid arthritis as evident by their elevated level of anti-CCP antibody in the plasma $(92.16 \pm 10.89 \mathrm{U} / \mathrm{mL})$. These 57 individuals-diagnosed as patients with RA also showed higher mean plasma level of rheumatoid factor 
(a)

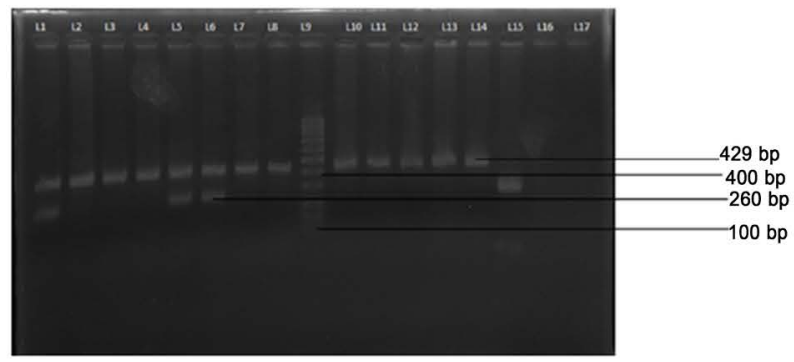

(c)

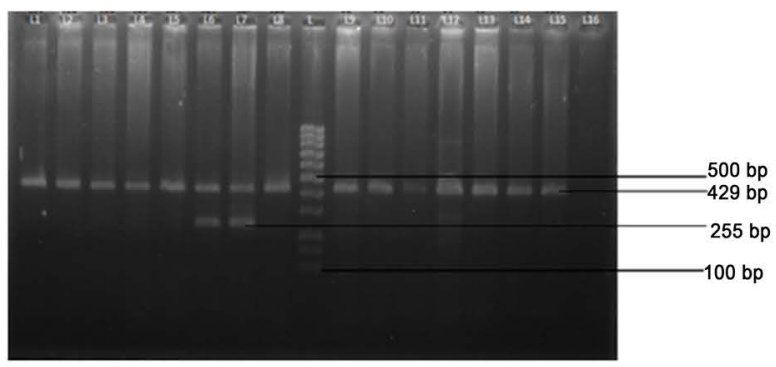

(e)

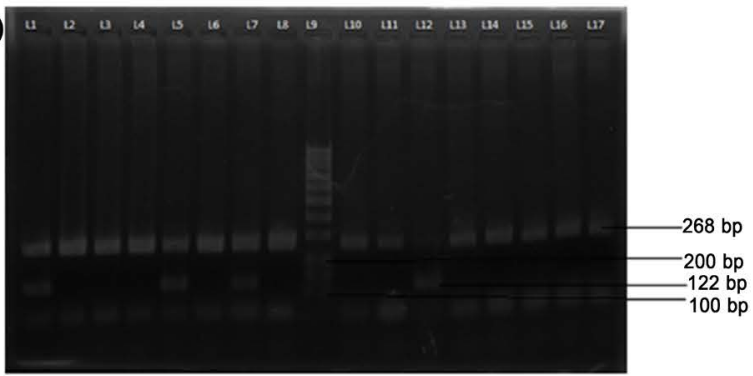

(b)

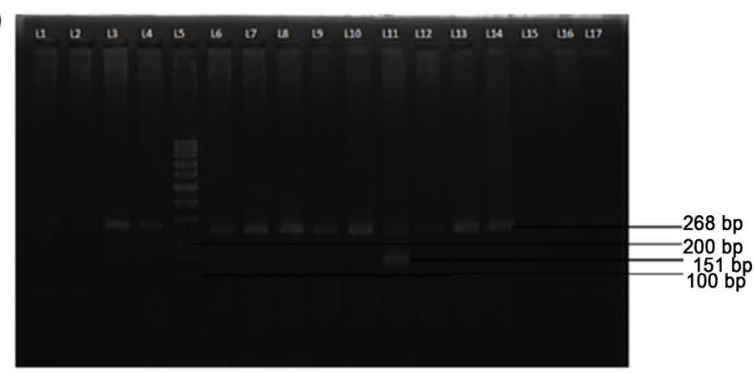

(d)

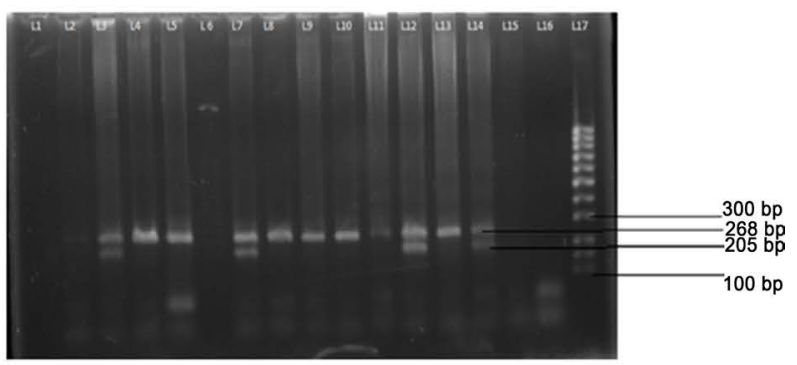

(f)

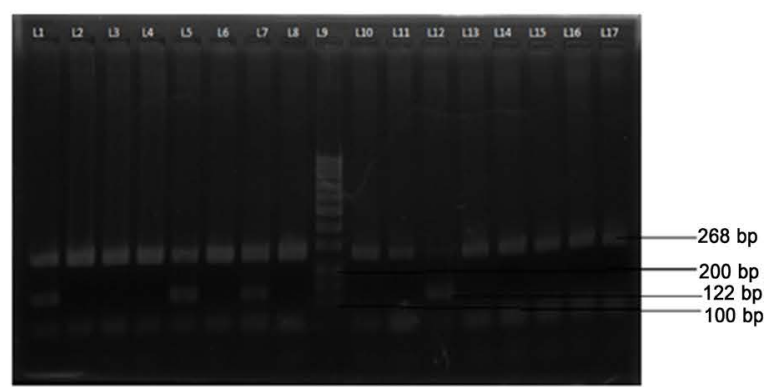

Figure 2. Agarose gel electrophoresis showing amplicons of HL-DRB and HLA-DQB genes. (a) Agarose gel electrophoresis showing the presence of HLA-DRB1 ${ }^{\star} 04$ gene. The presence of $260 \mathrm{bp}$ bands at lanes 1, 5, 6 and 15 indicated that respective individuals were HLA-DRB1 ${ }^{\star} 04$ positive. Amplicon with band size $429 \mathrm{bp}$ was for human growth hormone which was used as internal positive control. (b) Agarose gel electrophoresis showing the presence of HLA-DRB1 ${ }^{\star} 03$ gene. The presence of amplicon with a band size $151 \mathrm{bp}$ indicated HLA-DRB1 ${ }^{\star} 03$ positive individual. $\beta$-globin gene with a band size of 268 bp was used as an internal control. A $100 \mathrm{bp}$ ladder was used as marker DNA. (c) Agarose gel electrophoresis showing the presence of HLA-DRB1*01 gene. The presence of each allele was determined by their corresponding band size. The band size was $255 \mathrm{bp}$. The bands at lanes 6 and 7 indicate the presence of HLA-DRB $1^{\star} 01$ in respective individuals. Amplicon with band size 429 bp was for human growth hormone which was used as internal positive control to document successful PCR amplification. (d) Agarose gel electrophoresis showing the presence of HLA-DQB1 ${ }^{\star} 0201$ gene. Lanes $2,6,11$ and 13 represent positive amplicons for HLA-DQB1*0201 with band size 205 bp. $\beta$-globin gene with a band size of 268 bp was used as an internal control. A 100 bp ladder was used as marker DNA. The bands at the bottom of the gel represent the primer dimer. (e) Agarose gel electrophoresis showing the presence of HLA-DRB1 ${ }^{\star} 07$ gene. Lanes 3 and 13 represent positive amplicons for HLA-DRB1 ${ }^{\star} 07$ with band size 232 bp $\beta$-globin gene with a band size of $268 \mathrm{bp}$ was used as an internal control. A $100 \mathrm{bp}$ ladder was used as marker DNA. (f) Agarose gel electrophoresis showing the presence of HLA-DQB1 ${ }^{\star} 0301 / 4$ gene. Lanes $1,5,7$ and 12 represent positive amplicons for HLA-DQB1 ${ }^{\star} 0301 / 4$ with band size $122 \mathrm{bp}$. $\beta$-globin gene with a band size of $268 \mathrm{bp}$ was used as an internal control. A $100 \mathrm{bp}$ ladder was used as marker DNA. The bands at the bottom of the gel represent the primer dimer.

(RF) $(104.6 \pm 22.29 \mathrm{U} / \mathrm{mL})$. The patients suffering from rheumatoid arthritis exhibited significantly higher anti-CCP antibody and RF level ( $\geq 10 \mathrm{IU} / \mathrm{mL})$ compared to normal individual (Figure 3(a) and Figure 3(b)). The mean of age of the control and the RA groups were $23.5 \pm 11.0$ and $50.67 \pm 14.29$, respectively. The anthropometric and demographic data of the healthy individuals as well as the potential RA patients are given in Table 2 . 
(a)

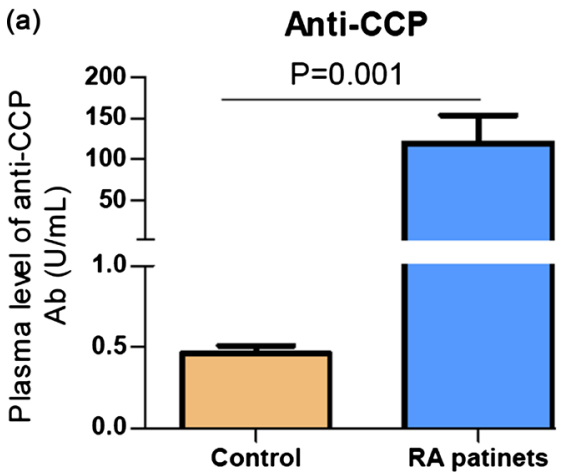

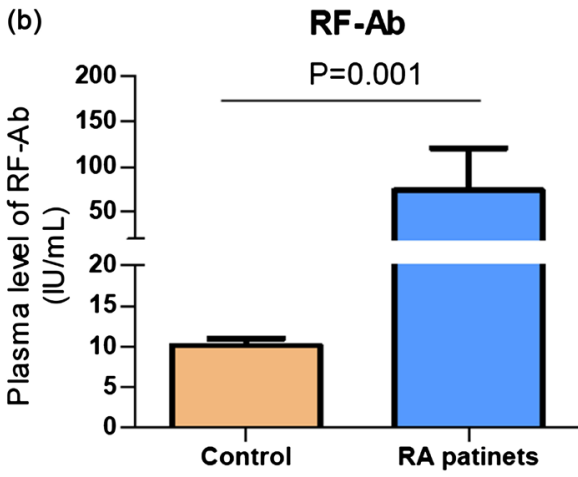

Figure 3. Plasma levels of anti-citrullinated peptide (anti-CCP) and rheumatoid factor (RF) antibody. (a) The level of anti-citrullinated peptide (anti-CCP) antibody (Ab) was measured by standard ELISA method and (b) The level of RF Ab was measured from the plasma samples of control and RA subjects. The mean levels of anti-CCP Ab $(\mathrm{I} / \mathrm{mL})$ and $\mathrm{RF}-\mathrm{Ab}(\mathrm{IU} / \mathrm{mL}$ ) of control (orange) and RA patients (blue) are indicated by Bars. The error bars represent the standard deviation (SD). P-values indicate the significance difference of anti-CCP Ab and RF-Ab levels between control and RA subjects.

Table 2. Demographic and anthropometric measurements of study participants.

\begin{tabular}{|c|c|c|}
\hline \multicolumn{3}{|c|}{ Healthy individual anthrometric and demographic data } \\
\hline \multirow{2}{*}{$\begin{array}{c}\text { Parameters } \\
\text { Gender }\end{array}$} & \multicolumn{2}{|c|}{ Control subjects $(n=34)$} \\
\hline & Male $(\mathrm{n}=18)$ & Female $(n=16)$ \\
\hline Age (years) & $23.5 \pm 11.0$ & $25.7 \pm 5.5$ \\
\hline Height $(\mathrm{cm})$ & $165.1 \pm 4.3$ & $153.6 \pm 9.1$ \\
\hline Weight (kg) & $63.2 \pm 8.5$ & $54.0 \pm 10.4$ \\
\hline BMI $\left(\mathrm{kg} / \mathrm{m}^{2}\right)$ & $23.2 \pm 2.7$ & $18.7 \pm 4.6$ \\
\hline Systolic blood pressure & $116.4 \pm 4.85$ & $114.4 \pm 5.8$ \\
\hline Diastolic blood pressure & $76.5 \pm 3.2$ & $77.8 \pm 4.7$ \\
\hline \multicolumn{3}{|c|}{ RA patients anthrometric and demographic data } \\
\hline Parameter & \multicolumn{2}{|c|}{ Patients $(\mathrm{n}=57)$} \\
\hline Gender & Male $(\mathrm{n}=30)$ & Female $(\mathrm{n}=27)$ \\
\hline Age (years) & $50.67 \pm 14.29$ & $42.38 \pm 11.40$ \\
\hline Height $(\mathrm{cm})$ & $158 \pm 5$ & $149.36 \pm 7.3$ \\
\hline Weight (kg) & $60.8 \pm 6.5$ & $48.0 \pm 7.5$ \\
\hline BMI $\left(\mathrm{kg} / \mathrm{m}^{2}\right)$ & $20.2 \pm 1.7$ & $17.7 \pm 3.3$ \\
\hline Systolic blood pressure & $118.4 \pm 3.4$ & $110.4 \pm 6.2$ \\
\hline Diastolic blood pressure & $74.5 \pm 4.2$ & $73.8 \pm 3.7$ \\
\hline
\end{tabular}

\subsection{Uric Acid, CRP, Glucose and Total Protein Levels in the Plasma of Study Participants}

The mean levels of plasma uric acid of healthy subjects and RA patients were $6.158 \pm 1.213$ and $6.293 \pm 0.9163(\mathrm{mg} / \mathrm{dL})$ respectively whereas, the mean plasma glucose levels of healthy subjects and RA patients were $105.9 \pm 4.82$ and $90.10 \pm$ 
$4.17(\mathrm{mg} / \mathrm{dL})$, respectively. Statistical analyses using unpaired t-test revealed that the levels of uric acid and glucose levels in the healthy individuals did not deviate significantly from that of RA patients.

On the contrary the mean plasma CRP level $(3.69 \pm 0.59 \mathrm{mg} / \mathrm{dL}$ for healthy control and $9.92 \pm 1.26 \mathrm{mg} / \mathrm{dL}$ for RA patients) and plasma total protein level (7.99 $\pm 0.26 \mathrm{mg} / \mathrm{dL}$ for healthy control and $6.80 \pm 0.14 \mathrm{mg} / \mathrm{dL}$ for RA patients) varied significantly between control group and RA patients as revealed by t-test. The data for biochemical parameters among the study participants are given in Table 3.

\subsection{Lipid Profile Analysis of the Study Participants}

The mean triglyceride (TG) levels in the plasma of healthy controls and RA patients were $112.2 \pm 8.82$ and $234.4 \pm 16.54 \mathrm{mg} / \mathrm{dL}$, respectively. Their mean plasma cholesterol levels were $116.4 \pm 6.98$ and $219.7 \pm 11.30 \mathrm{mg} / \mathrm{dL}$, respectively. In both cases significant variation was observed between healthy subjects and RA patients. To further evaluate the lipid profile, HDL- and LDL-cholesterol levels were measured. The mean plasma HDL cholesterol in RA patients $(36.45 \pm$ $6.5 \mathrm{mg} / \mathrm{dL})$ was significantly lower compared to that of healthy controls (42.58 \pm $9.3 \mathrm{mg} / \mathrm{dL}$ ). Unlike HDL-cholesterol, determination of LDL-cholesterol level did not exhibit any significant difference between healthy controls (125.69 \pm 11.6 $\mathrm{mg} / \mathrm{dL})$ and RA patients $(132.5 \pm 5.8 \mathrm{mg} / \mathrm{dL})$. Data have been presented in Table 4.

\subsection{Activities of Aspartate Aminotransferase, Alanine Aminotransferase and Alkaline Phosphatase in the Plasma of the Healthy Controls and RA Patients}

The activity of AST for the healthy individuals and RA patients showed significant deviation upon statistical analysis. The mean value for controls was $6.50 \pm$

Table 3. Levels of uric acid, CRP, glucose and total protein in the plasma of study participants.

\begin{tabular}{ccccc}
\hline Study subjects & $\begin{array}{c}\text { Uric acid } \\
(\mathrm{mg} / \mathrm{dL})\end{array}$ & $\begin{array}{c}\text { CRP } \\
(\mathrm{mg} / \mathrm{dL})\end{array}$ & $\begin{array}{c}\text { Glucose } \\
(\mathrm{mg} / \mathrm{dL})\end{array}$ & $\begin{array}{c}\text { Total protein } \\
(\mathrm{mg} / \mathrm{dL})\end{array}$ \\
\hline $\begin{array}{c}\text { Healthy controls }(\mathrm{n}=34) \\
\text { RA patients }(\mathrm{n}=58)\end{array}$ & $6.158 \pm 1.213$ & $3.692 \pm 0.5816$ & $105.9 \pm 4.82$ & $7.99 \pm 0.26$ \\
$\begin{array}{c}\text { Statistics between control } \\
\text { and patient group }\end{array}$ & $\mathrm{P}=0.9302$ & $\mathrm{P}=0.0001$ & $\mathrm{P}=0.186$ & $\mathrm{P}=0.06$ \\
\hline
\end{tabular}

Table 4. Aspartate/Alanine aminotransferase and Alkaline phosphatase activities in the plasma of the healthy controls and RA patients.

\begin{tabular}{ccccc}
\hline Study subjects & $\begin{array}{c}\text { Triglyceride level } \\
(\mathrm{mg} / \mathrm{dL})\end{array}$ & $\begin{array}{c}\text { Cholesterol level } \\
(\mathrm{mg} / \mathrm{dL})\end{array}$ & $\begin{array}{c}\text { HDL level } \\
(\mathrm{mg} / \mathrm{dL})\end{array}$ & $\begin{array}{c}\text { LDLlevel } \\
(\mathrm{mg} / \mathrm{dL})\end{array}$ \\
\hline Healthy controls $(\mathrm{n}=34)$ & $112.2 \pm 8.82$ & $161.4 \pm 6.98$ & $42.58 \pm 9.3$ & $125.69 \pm 11.6$ \\
RA patients $(\mathrm{n}=58)$ & $234.4 \pm 16.54$ & $219.7 \pm 11.3$ & $36.45 \pm 6.5$ & $132.5 \pm 5.8$ \\
$\begin{array}{c}\text { Statistics between control } \\
\text { and patient group }\end{array}$ & $\mathrm{P}=0.0001$ & $\mathrm{P}=0.0004$ & $\mathrm{P}=0.035$ & $\mathrm{P}=0.07$ \\
\hline
\end{tabular}


$0.98 \mathrm{U} / \mathrm{L}$, while for RA patents this value was $14.16 \pm 0.86 \mathrm{U} / \mathrm{L}$, which varied significantly ( $\mathrm{p}<0.001$; Figure $4(\mathrm{a})$ ). The mean level of ALT in the plasma of healthy controls was $6.809 \pm 0.98 \mathrm{U} / \mathrm{L}$, and for patients, the value was $10.46 \pm$ $0.8397 \mathrm{U} / \mathrm{L}$. Statistical analyses revealed a significantly increased ALT level in the RA patients in comparison to that of healthy individuals (Figure 4(b)).

In addition, measurement of Alkaline phosphatase (ALP) levels for controls and RA patients revealed the mean ALP levels for control and RA patients were $86.72 \pm 6.56$ and $33.67 \pm 2.53 \mathrm{U} / \mathrm{L}$, respectively. Interestingly unlike ALT and AST, the ALP levels exhibited the opposite trend as it was observed to be significantly lower in the RA patients compared to the healthy individuals (Figure 4(c)).

\subsection{Genotypic Distribution of HLA-DRB1*01, HLA-DRB1*03, HLA-DRB1*04, HLA-DRB1*07, HLA-DQB1*0201 and HLA-DQB1*0301/4 among the Study Subjects}

To investigate the association of HLA-alleles with RA phenotype, it is important
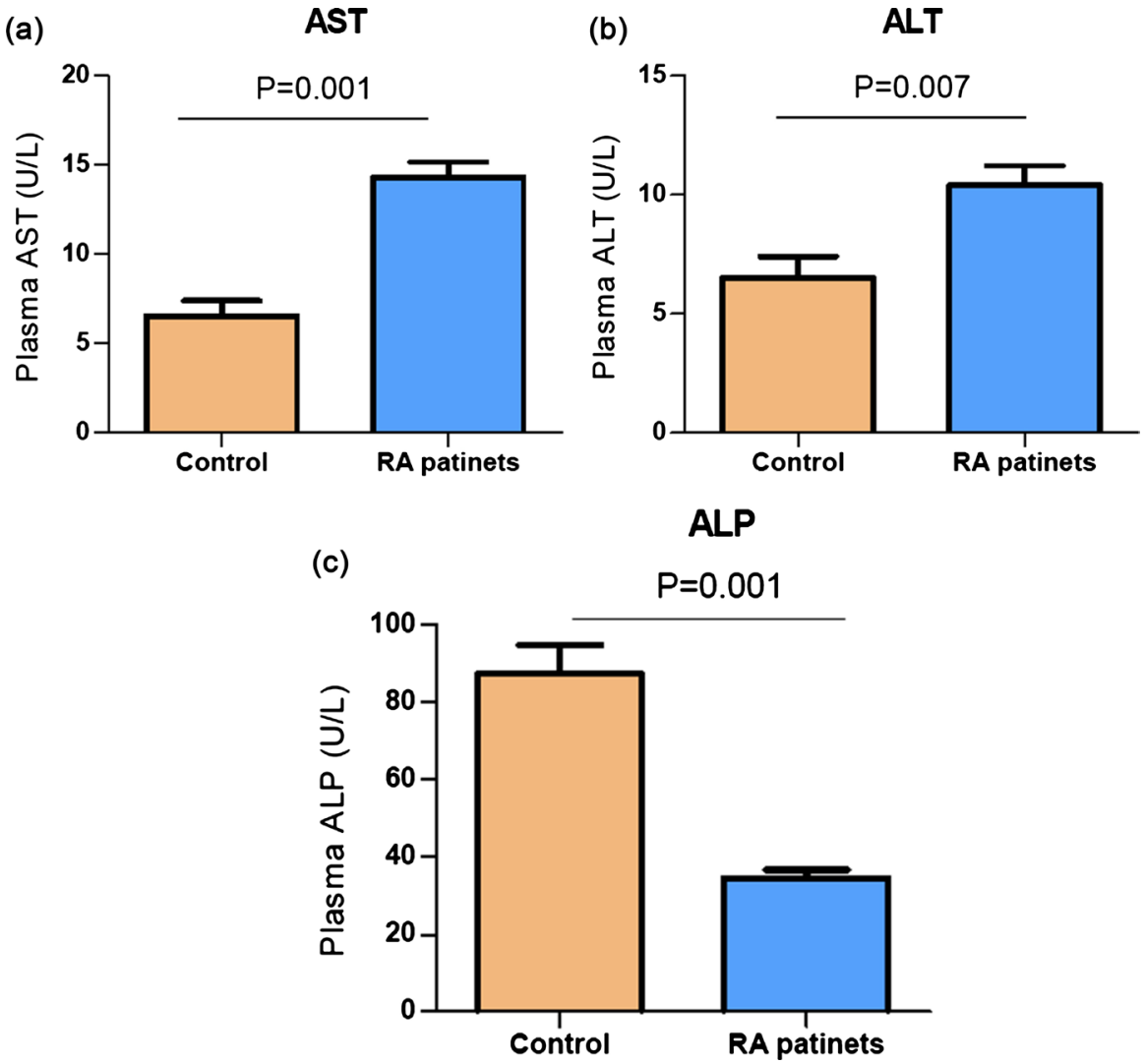

Figure 4. Aspartate/Alanine-aminotransferase and Alkaline phosphatase levels in the plasma of the healthy controls and samples. (a) The levels of Aspartate amino transferase (AST), (b) alanine amino transferase (ALT) and (c) alkaline phosphatase (ALP) were measured from the plasma samples of controls and RA subjects by standard biochemical methods. The mean AST, ALT and ALP levels (U/L) of control (orange) and RA patients (blue) are indicated by Bars. The error bars represent the standard deviation (SD). P-value indicates the significance difference of AST, ALT and ALP levels between control and RA subjects. 
to identify the HLA-alleles with higher frequency of occurrence within the population under investigation. In order to identify the HLA-allele frequency, we systematically mined the Allele Frequency Net Database

(http://www.allelefrequencies.net/default.asp) with a special emphasis on SouthEast Asian population. Analysis revealed that HLADRB1 ${ }^{\star} 04$ allele has the relatively higher frequency (13\%) in South-East Asian population. Based on the frequency analysis we selected six different HLA alleles-HLA-DRB ${ }^{\star} 01$, HLADRB $1{ }^{*} 03, \mathrm{HLA}-\mathrm{DRB} 1{ }^{*} 04, \mathrm{HLA}-\mathrm{DRB} 1^{\star} 07, \mathrm{HLA}-\mathrm{DQB} 1^{\star} 0201$ and HLA-DQB1 ${ }^{\star} 0301 / 4$. Figure 5 (a) shows the frequency (\%) of occurrence of the respective HLA-genotype among healthy controls and RA subjects. Among the analyzed alleles, the highest number of RA patients $(n=20)(34 \%)$ were positive for
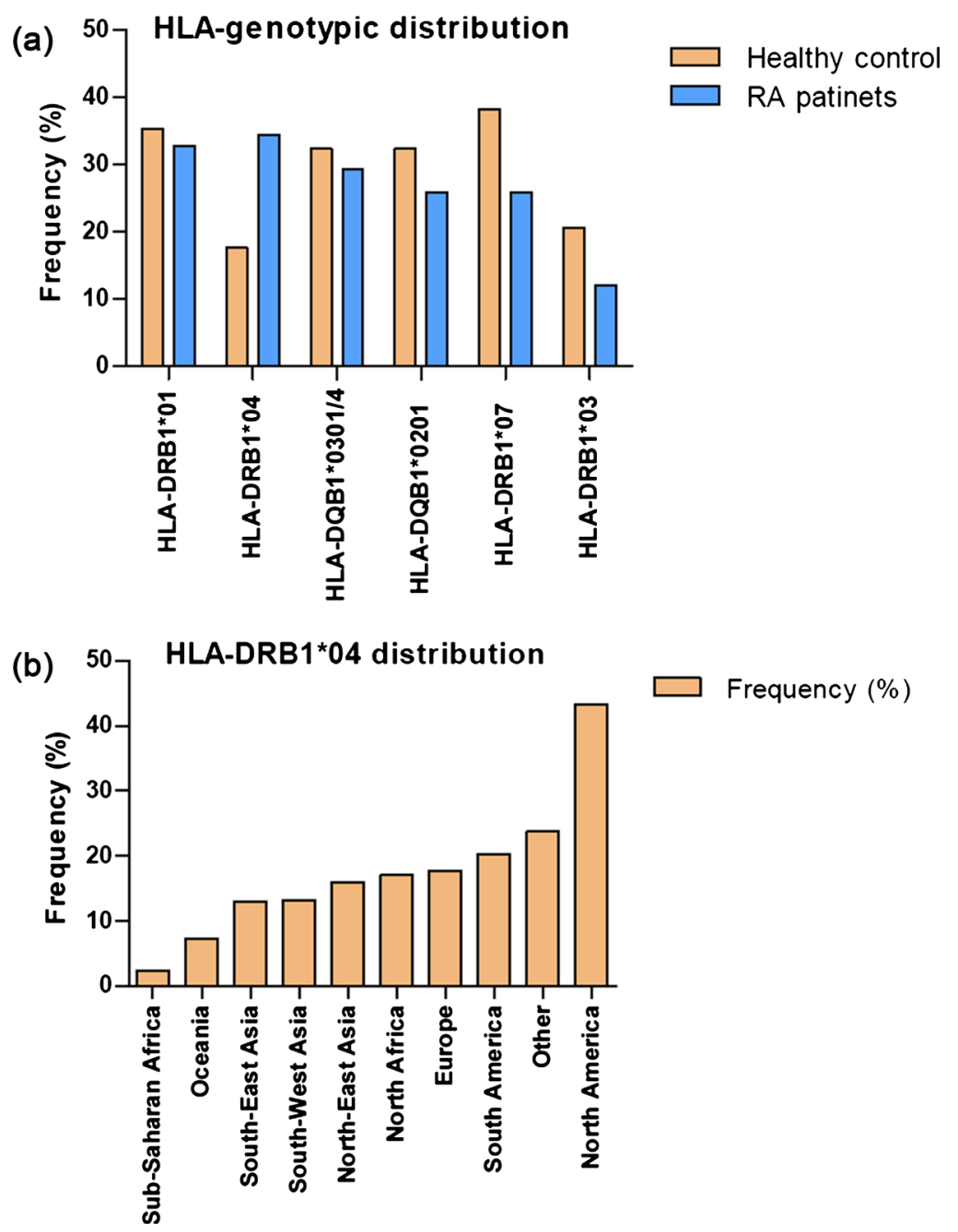

Figure 5. HLA-allele frequencies in RA patients and world-wide population. (a) Allele frequency for HLA-DRB ${ }^{\star} 01$, HLA-DRB $1^{*} 03$, HLA-DRB $1^{*} 04$, HLA-DRB $1^{\star} 07$, HLADQB1*0201 and HLA-DQB1*0301/4 in control and RA subjects are shown as bar diagram. Allele frequency was calculated as $\%$ of alleles found positive in particular group (control or RA). (b) Allele frequency of HLA-DRB1 ${ }^{*} 04$ in world-wide population is shown. Allele frequency is represented by the $\%$ of occurrence of HLA-DRB1 ${ }^{\star} 04$ in the population of a particular geographic location. 
HLA-DRB1 ${ }^{\star} 04$ allele followed by HLA-DRB1*01 (32\%) and HLA-DQB1 ${ }^{\star} 0301 / 4$ (29\%). For both HLA-DRB1 ${ }^{\star} 07$ and HLA-DQB1 ${ }^{\star} 0201$ fifteen RA patients $(26 \%)$ were found be positive in each case. The lowest distribution frequency was evident for HLA-DRB ${ }^{\star} 03$ as only seven individuals were found to be positive out of $57 \mathrm{RA}$ patients. Since HLA-DRB $1^{\star} 04$ allele demonstrated the highest frequency in the RA subjects we sought to investigate its frequency in world-wide population. Mining and analysis of allele frequency data for HLA-DRB ${ }^{\star} 04$ showed an interesting phenomenon. Highest frequency for this HLA allele was observed for United States (Figure 5(b)).

\subsection{Distribution of Anti Cyclic Citrullinated Peptide and Rheumatoid Factor Antibodies among the Study Subjects}

Subsequently we sought to identify the correlation between patient-specific HLAallele occurrence and clinical parameters such as serum anti-CCP and RF antibody levels. Result revealed a nearly uniform distribution of anti-CCP antibody among RA patients with different HLA-genotype (HLA-DRB1*01, HLA-DRB1*03, HLADRB1 ${ }^{\star} 04, \mathrm{HLA}-\mathrm{DRB} 1{ }^{*} 07, \mathrm{HLA}-\mathrm{DQB} 1{ }^{*} 0201$ and HLA-DQB1*0301/4) (Figure 6(a)). The highest levels of anti-CCP antibody was observed for the RA patients with HLA-DRB $1^{\star} 04$ allele. Intriguingly, in case of levels of RF antibody a diverse distribution pattern across RA patients having different HLA alleles emerged (Figure 6(b)). For instance, the mean level of rheumatoid factor antibody $(\mathrm{U} / \mathrm{mL})$ was found to be significantly higher in the RA patients having HLA-DRB1*04 allele compared to RA patients with other HLA alleles (HLA-DRB1*01, HLADQB1 ${ }^{\star} 0301 / 4$, HLA-DRB1 ${ }^{\star} 07$ and HLA-DQB1 $\left.{ }^{\star} 0201\right)$. We performed student's t-test to estimate the level of significance of anti-CCP (Figure 6(a)) and RF (Figure 6(b)) between control and RA patients pre-grouped by their HLA genotypes. The results showed that for all tested HLA alleles the levels of anti-CCP (Figure 6(a)) and RF (Figure 6(b)) varies significantly between control and RA patients where RA patients with different HLA genotypes exhibited significantly higher anti-CCP and RF levels compared to the control subjects. Additionally, we performed student's t test to identify whether the anti-CCP and RF levels were significantly different among RA patients with different HLA genotypes. Since RA patients with HLA DRB1 ${ }^{\star} 04$ exhibited the highest levels of anti-CCP and RF levels compared to the RA patients with other HLA genotype, t-tests were performed in a pair wise manner, where the anti-CCP (Figure 6(a)) and RF (Figure 6(b)) levels were compared between HLA DRB1*04 and rest of HLA alleles. The results showed that, the level of Anti-CCP and RF were significantly higher in RA patients with HLA $\mathrm{DRB} 1^{\star} 04$ compared to those having different HLA genotypes. These findings underscore a potential association between HLA genotype with clinical markers of RA such as RF which can be regarded as indicators of severity of the diseases. Interestingly, for the healthy controls the RF levels remained constant across all individual harboring different HLA alleles including HLA-DRB1*04. 


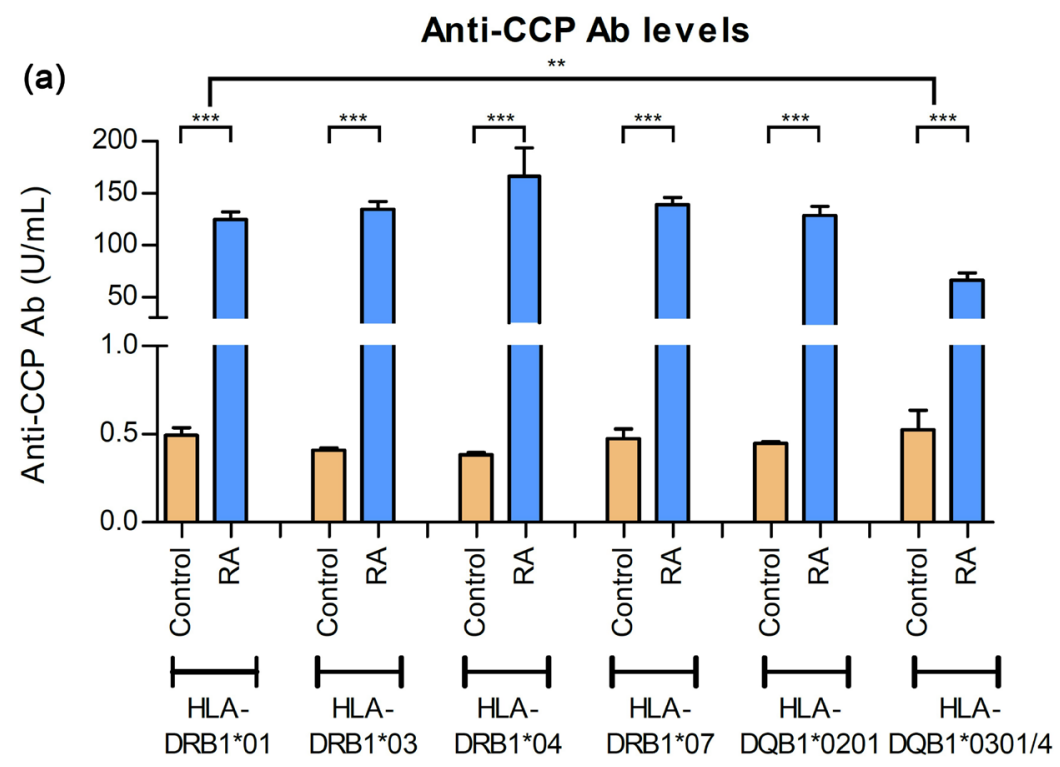

(b)

RF Ab levels

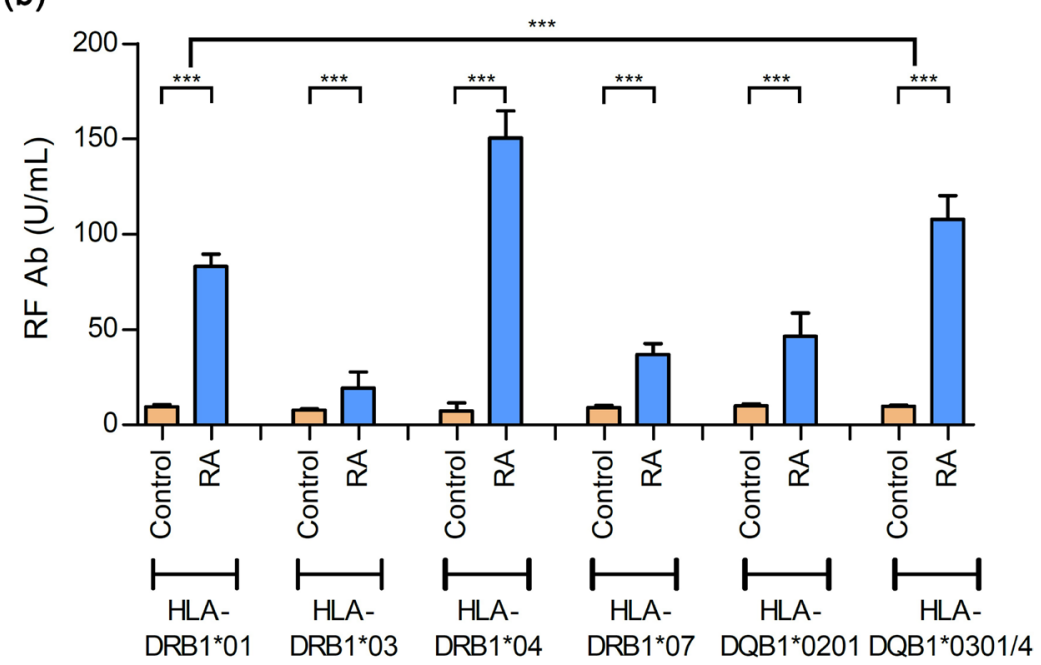

Figure 6. Levels of anti-cyclic citrullinated peptide (anti-CCP) and rheumatoid factor (RF) antibodies in RA patients measured among different HLA alleles. (a) Distribution of the level of plasma anti-CCP antibody among different genotypes of healthy controls and patients with rheumatoid arthritis. (b) Distribution of the level of plasma RF antibody among different genotypes of healthy controls and patients with rheumatoid arthritis. Student t-tests were carried out to estimate the level of significance. P-values are indicated by star signs, where ${ }^{\star * \star}$ indicates $\mathrm{p}$-value of less than 0.001 , whereas ${ }^{\star *}$ indicates $\mathrm{p}$-value of less than 0.05 .

The preferential occurrence of $\mathrm{HLA}-\mathrm{DRB} 1^{\star} 04$ allele in the RA patients with high RF value may indicate susceptibility of the individuals possessing this particular allele to have higher RF levels if they are diagnosed with RA in Bangladeshi population.

\section{Discussion}

The current study investigated HLA-allele distribution in 57 rheumatoid arthri- 
tis patients and 34 healthy control subjects with reference to Bangladeshi population under the assumption that HLA-allele frequency analysis may reveal the genetic predisposition indicating the propensity of rheumatoid arthritis. Various clinical parameters of RA such as Anti-CCP and RF were measured to confirm the diseases as well as to estimate the severity of the RA. The striking association between a specific HLA alleles-HLA-DRB $1^{\star} 04$ with high levels of RF underscored the importance of HLA-genotyping as a predictor of genetic predisposition for the severity of the RA. A plethora of studies have shown a possible linkage between the presence of certain HLA alleles and the occurrence of this autoimmune disease [5] [17] [18] [19]. In light of these findings we propose the association of HLA-DRB ${ }^{\star} 04$ allele with the elevated RF levels in the patients with rheumatoid arthritis. The current study includes, apart from the clinical parameters of RA, such as Anti-CCP and RF, various patients' related anthropometric factors such as age, sex, systolic and diastolic blood pressure, to establish a connection of HLA-allele predisposition and clinical metadata.

The association of various biochemical parameters, e.g. uric acid, C-reactive protein (CRP), glucose and total protein levels, with RA has been reported. For example patients with progressive RA are considered to be at risk of having high serum uric acid [20], CRP levels [21] and abnormal glucose metabolism [22]. In line with these findings we also observed high CRP levels in RA patients. CRP is an effective marker of systemic inflammation and considered as the potent biomarker for the onset of cardiovascular disease associated with RA [23]. However, no significant elevation for serum uric acid, glucose and total protein levels was observed in RA patients in comparison with normal subjects. The measurement of lipid profiles of the study subjects revealed a positive correlation of dyslipidemia and RA since, the RA patients exhibited significantly higher levels of TG, total cholesterol and lower levels of HDL compared to the healthy counterparts. Previously it was reported that RA patients have a higher risk of developing pre-mature cardiovascular disease as evident by their lower HDL cholesterol levels [24]. Another study conducted by Alizade et al. demonstrated the association of RF levels with the extent and severity of coronary artery disease [25]. The risk of cardiovascular disease in RA patients also emerges from the inability of RA patients to manage the risk through exercise. Therefore, it is of utmost importance to monitor the lipid profile in RA patients followed by intervention to minimize the long-term risk of developing cardiovascular diseases. The higher levels of AST and ALT in the RA patients than the healthy controls can be attributed to the medication as part of initiating disease-modifying antirheumatic drugs (DMARD) therapy that the patients were receiving through the study period. The hepatocoxic side-effects of rheumatoid arthiritis drugs such as methotrexate (MTX), leflunomide (LEF) have been reported earlier [26]. These drugs, due to their cytotoxic effects, were deemed responsible for the elevated levels of AST and ALT in RA patients who receive these drugs [26]. In contrast to AST and ALT, intriguingly, ALP level in RA patients was found to be lower compared 
to healthy individuals. Earlier studies have found the higher levels of ALP in RA patients although the underlying etiology has not been well understood [27].

In order to establish a link between HLA genotyping and clinical parameters of rheumatoid arthritis-anti-CCP and RF antibody, six different HLA alleles (HLA-DRB1 ${ }^{\star} 01$, HLA-DRB1 ${ }^{\star} 03$, HLA-DRB1 ${ }^{\star} 04$, HLA-DRB1 ${ }^{\star} 07$, HLA-DQB1 ${ }^{\star} 0201$ and HLA-DQB1 $\left.{ }^{\star} 0301 / 4\right)$ were analyzed among the study subjects. The selection of these alleles was based on the systematic analysis of the allele frequency of different HLA alleles in South-East Asian population. Based on the frequency analysis, we selected these six different HLA alleles that represent South-East Asian population diversity with respect to HLA-alleles. Analyses of the genotypic distribution of HLA genes in the study subjects indicated that the alleles were widely distributed throughout healthy subjects and RA patients. An earlier study reported a possible association of HLA-DRB1 ${ }^{\star} 01$ allele with rheumatoid arthritis in populations representing a wide variety of ethnicity and geographical locations [28] [29] [30]. Another study showed the association of the propensity of RA with HLA-DRB1*04 in Chinese population [31]. Furthermore, study with RA patients among Syrian population was found to be strongly associated with both HLA-DRB1 ${ }^{\star} 04$ and HLA-DRB1*01 [32]. However, no such association has been shown for South-East Asian population. North American Rheumatoid Arthritis Consortium, performed a study involving a large cohort of study-subjects of the North American origin, have uncovered a significant association between the susceptibility of RA and HLA-DRB1*04 [33].

In our study the frequency of RA patients harboring HLA-DRB1 ${ }^{\star} 04$ allele (34\%) was found to be double than that of in healthy individuals (17\%) indicating the frequency occurrence of this particular allele is relatively higher in RA patients.

Next we asked whether an association could be established between HLA alleles and RA specific clinical parameters. To achieve this, we first divided the study subjects including the healthy controls and RA patients into different groups according to the presence of distinct HLA alleles and then, the levels of anti-CCP and RF antibodies were compared between the groups. No notable pattern was observed with respect to distribution of anti-CCP antibody levels among the different HLA-groups (Figure 6(a)) though HLA-DRB1*04 positive RA patients had the highest and HLADQB1*0301/4 positive patients had the lowest levels. Surprisingly, for RF antibody a strong pattern emerges, which indicated that the RA patients carrying HLA-DRB ${ }^{\star} 04$ allele exhibited significantly higher levels of RF antibody compared to the RA patients having other HLA-alleles. This striking finding indicated that HLA-genotyping has the potential not only to estimate the propensity of RA but also can be utilized to RA progression and severity. Previously, studies have established a potential link between the RF and with disease activity in rheumatoid arthritis [34].

The scarcity of genotypic studies of South-East Asian population, has posed a major challenge in the estimation of the percentage of population who are at risk 
of rheumatoid arthritis. The current study can be basis of a large-scale study in the region to uncover the population-wide genotypic distribution of HLA alleles associated with RA.

\section{Acknowledgements}

This study was supported in part by the Grants provided by the University Grants Commission to University of Dhaka, Bangladesh. We express our heartfelt gratitude to the participants who willingly donated their blood for this study.

\section{Conflicts of Interest}

The authors declare no conflicts of interest regarding the publication of this paper.

\section{References}

[1] Kurko, J., et al. (2013) Genetics of Rheumatoid Arthritis-A Comprehensive Review. Clinical Reviews in Allergy \& Immunology, 45, 170-179.

https://doi.org/10.1007/s12016-012-8346-7

[2] MacGregor, A.J., et al. (2000) Characterizing the Quantitative Genetic Contribution to Rheumatoid Arthritis Using Data from Twins. Arthritis \& Rheumatology, 43, 30-37. https://doi.org/10.1002/1529-0131(200001)43:1<30::AID-ANR5>3.0.CO;2-B

[3] van der Helm-van Mil, A.H., Wesoly, J.Z. and Huizinga, T.W. (2005) Understanding the Genetic Contribution to Rheumatoid Arthritis. Current Opinion in Rheumatology, 17, 299-304. https://doi.org/10.1097/01.bor.0000160780.13012.be

[4] Lee, H.S., et al. (2007) Interaction between Smoking, the Shared Epitope, and Anti-Cyclic Citrullinated Peptide: A Mixed Picture in Three Large North American Rheumatoid Arthritis Cohorts. Arthritis \& Rheumatology, 56, 1745-1753.

https://doi.org/10.1002/art.22703

[5] Newton, J.L., Harney, S.M.J., Wordsworth, B.P. and Brown, M.A. (2004) A Review of the MHC Genetics of Rheumatoid Arthritis. Genes \& Immunity, 5, 151-157. https://doi.org/10.1038/sj.gene.6364045

[6] Weyand, C.M. and Goronzy, J.J. (2000) Association of MHC and Rheumatoid Arthritis. HLA Polymorphisms in Phenotypic Variants of Rheumatoid Arthritis. Arthritis Research \& Therapy, 2, 212-216. https://doi.org/10.1186/ar90

[7] Mahtani, K.R., et al. (2013) Autoimmune Markers for the Diagnosis of Rheumatoid Arthritis in Primary Care: Primary Care Diagnostic Technology Update. British Journal of General Practice, 63, 553-554. https://doi.org/10.3399/bjgp13X673919

[8] Palazzi, C., et al. (2012) Autoantibodies in Patients with Chronic Hepatitis C Virus Infection: Pitfalls for the Diagnosis of Rheumatic Diseases. Autoimmunity Reviews, 11, 659-663. https://doi.org/10.1016/j.autrev.2011.11.011

[9] Schellekens, G.A., de Jong, B.A., van den Hoogen, F.H., van de Putte, L.B. and van Venrooij, W.J. (1998) Citrulline Is an Essential Constituent of Antigenic Determinants Recognized by Rheumatoid Arthritis-Specific Autoantibodies. The Journal of Clinical Investigation, 101, 273-281. https://doi.org/10.1172/JCI1316

[10] Steuer, A., Watkins, J., Smith, F., Day, L., Demetriadi, F. and Chapel, H. (2008) RF Latex and Anti-CCP Antibodies: A Combined Strategy for Diagnosing RA in Primary Care? Rheumatology (Oxford), 47, 375-376.

https://doi.org/10.1093/rheumatology/kem367 
[11] Gavrila, B.I., Ciofu, C. and Stoica, V. (2016) Biomarkers in Rheumatoid Arthritis, What Is New? Journal of Medicine and Life, 9, 144-148.

[12] Nielsen, S.F., et al. (2012) Elevated Rheumatoid Factor and Long Term Risk of Rheumatoid Arthritis: A Prospective Cohort Study. BMJ, 345, e5244. https://doi.org/10.1136/bmj.e5244

[13] Paimela, L., Palosuo, T., Leirisalo-Repo, M., Helve, T. and Aho, K. (1995) Prognostic Value of Quantitative Measurement of Rheumatoid Factor in Early Rheumatoid Arthritis. British Journal of Rheumatology, 34, 1146-1150. https://doi.org/10.1093/rheumatology/34.12.1146

[14] Weyand, C.M. and Goronzy, J.J. (1994) HLA-DRB1 Alleles as Severity Markers in RA. Bulletin on the Rheumatic Diseases, 43, 5-8.

[15] Seidl, C., et al. (1997) HLA-DRB1 04 Subtypes Are Associated with Increased Inflammatory Activity in Early Rheumatoid Arthritis. British Journal of Rheumatology, 36, 941-944. https://doi.org/10.1093/rheumatology/36.9.941

[16] Afruza, R., et al. (2014) Renin Gene Polymorphisms in Bangladeshi Hypertensive Population. Journal of Genomics, 2, 45-53. https://doi.org/10.7150/jgen.5193

[17] Onuora, S. (2015) Rheumatoid Arthritis: Clues to the HLA-RA Connection from T-Cell Cross-Reactivity to Vinculin and Microorganisms. Nature Reviews Rheumatology, 11, 384. https://doi.org/10.1038/nrrheum.2015.73

[18] Kim, K., Bang, S.-Y., Lee, H.-S. and Bae, S.-C. (2017) Update on the Genetic Architecture of Rheumatoid Arthritis. Nature Reviews Rheumatology, 13, 13-24. https://doi.org/10.1038/nrrheum.2016.176

[19] Dendrou, C.A., Petersen, J., Rossjohn, J. and Fugger, L. (2018) HLA Variation and Disease. Nature Reviews Immunology, 18, 325-339.

https://doi.org/10.1038/nri.2017.143

[20] Magnus, J.H., Doyle, M.K. and Srivastav, S.K. (2010) Serum Uric Acid and Self-Reported Rheumatoid Arthritis in a Multiethnic Adult Female Population. Current Medical Research and Opinion, 26, 2157-2163. https://doi.org/10.1185/03007995.2010.502007

[21] Amos, R.S., Constable, T.J., Crockson, R.A., Crockson, A.P. and McConkey, B. (1977) Rheumatoid Arthritis: Relation of Serum C-Reactive Protein and Erythrocyte Sedimentation Rates to Radiographic Changes. British Medical Journal, 1, 195-197. https://doi.org/10.1136/bmj.1.6055.195

[22] Pi, H., et al. (2017) Abnormal Glucose Metabolism in Rheumatoid Arthritis. BioMed Research International, 2017, Article ID: 9670434. https://doi.org/10.1155/2017/9670434

[23] Graf, J., Scherzer, R., Grunfeld, C. and Imbode, J. (2009) Levels of C-Reactive Protein Associated with High and Very High Cardiovascular Risk Are Prevalent in $\mathrm{Pa}$ tients with Rheumatoid Arthritis. PLoS ONE, 4, e6242. https://doi.org/10.1371/journal.pone.0006242

[24] Steiner, G. and Urowitz, M.B. (2009) Lipid Profiles in Patients with Rheumatoid Arthritis: Mechanisms and the Impact of Treatment. Seminars in Arthritis and Rheumatism, 38, 372-381. https://doi.org/10.1016/j.semarthrit.2008.01.015

[25] Alizade, E., et al. (2015) The Relationship between Rheumatoid Factor Levels and Coronary Artery Lesion Complexity and Severity in Patients with Stable Coronary Artery Disease. Postępy w Kardiologii Interwencyjnej, 11, 26-31. https://doi.org/10.5114/pwki.2015.49181

[26] Curtis, J.R., et al. (2010) Elevated Liver Enzyme Tests among Patients with Rheumatoid Arthritis or Psoriatic Arthritis Treated with Methotrexate and/or Leflunomide. 
Annals of the Rheumatic Diseases, 69, 43-47. https://doi.org/10.1136/ard.2008.101378

[27] Nanke, Y., Kotake, S., Akama, H. and Kamatani, N. (2002) Alkaline Phosphatase in Rheumatoid Arthritis Patients: Possible Contribution of Bone-Type ALP to the Raised Activities of ALP in Rheumatoid Arthritis Patients. Clinical Rheumatology, 21, 198-202. https://doi.org/10.1007/s10067-002-8285-4

[28] Sandoughi, M., et al. (2011) Frequency of HLA-DRB1 Alleles in Rheumatoid Arthritis Patients in Zahedan, Southeast Iran. Annals of Saudi Medicine, 31, 171-173. https://doi.org/10.4103/0256-4947.78205

[29] Gonzalez-Juanatey, C., et al. (2003) HLA-DRB1 Status Affects Endothelial Function in Treated Patients with Rheumatoid Arthritis. The American Journal of Medicine, 114, 647-652. https://doi.org/10.1016/S0002-9343(03)00133-5

[30] Kerlan-Candon, S., Combe, B., Vincent, R., Clot, J., Pinet, V. and Eliaou, J.-F. (2001) HLA-DRB1 Gene Transcripts in Rheumatoid Arthritis. Clinical \& Experimental Immunology, 124, 142-149. https://doi.org/10.1046/j.1365-2249.2001.01498.x

[31] Yang, M., et al. (2013) Meta-Analysis of the Association of HLA-DRB1 with Rheumatoid Arthritis in Chinese Populations. BMC Musculoskeletal Disorders, 14, 307. https://doi.org/10.1186/1471-2474-14-307

[32] Mourad, J. and Monem, F. (2013) HLA-DRB1 Allele Association with Rheumatoid Arthritis Susceptibility and Severity in Syria. Revista Brasileira de Reumatologia, 53, 47-56. https://doi.org/10.1016/S2255-5021(13)70005-1

[33] Guthrie, K.A., Tishkevich, N.R. and Nelson, J.L. (2009) Non-Inherited Maternal Human Leukocyte Antigen Alleles in Susceptibility to Familial Rheumatoid Arthritis. Annals of the Rheumatic Diseases, 68, 107-109. https://doi.org/10.1136/ard.2008.092312

[34] Choe, J.Y., et al. (2013) Relation of Rheumatoid Factor and Anti-Cyclic Citrullinated Peptide Antibody with Disease Activity in Rheumatoid Arthritis: Cross-Sectional Study. Rheumatology International, 33, 2373-2379.

https://doi.org/10.1007/s00296-013-2734-Z 